\title{
A link between cytotoxicity in cell culture and gastrointestinal side effects of oral anticoagulants: bench-to-bedside
}

\author{
Kubat $\mathrm{E}^{1,2}$, Gurpinar $\mathrm{OA}^{2}$, Karasoy $\mathrm{D}^{3}$, Onur $\mathrm{MA}^{2}$ \\ Department of Cardiovascular Surgery, Gülhane Training and Research Hospital, Ankara, Turkey. \\ ekubat@gmail.com
}

\begin{abstract}
OBJECTIVE: Warfarin and nonvitamin-K oral anticoagulants including dabigatran, rivaroxaban, and apixaban are commonly used in the prophylaxis and treatment of systemic embolism and deep vein thrombosis. In this study, we aimed to compare the cytotoxic effects of warfarin and new oral anticoagulants and to show a possible correlation between cell cytotoxicity and gastrointestinal side effects in the real-life setting.

METHODS: L929 cells were incubated with test materials. At 24 and 48 hours, morphological changes and cell viability were evaluated.

RESULTS: At 24 and 48 hours, dabigatran resulted in altered cell morphology in all dilutions, while rivaroxaban, apixaban, and warfarin showed similar morphology with the control group, except for dilution I. Dabigatran and warfarin at 24 hours and at 48 hours had a statistically significantly lower cell viability in all dilutions, compared to the control group.

CONCLUSION: Gastrointestinal side effect profiles of these four agents in a real-life setting is consistent with the results obtained from the present study. There is no sole factor with the potential of explaining the entire gastrointestinal side effect profiles of anticoagulant agents. However, direct cytotoxic effects of anticoagulants should be considered primarily for gastrointestinal side effects in accordance with the results of present headto-head cytotoxicity study (Tab. 5, Fig. 3, Ref. 28). Text in PDF www.elis.sk. KEY WORDS: dabigatran, rivaroxaban, apixaban, warfarin, cell viability.
\end{abstract}

\section{Introduction}

Various anticoagulants have been used for several cardiovascular diseases including the prevention of cardiac thromboembolism in patients with atrial fibrillation, mechanical heart valves, and acute myocardial infarction, as well as treatment or secondary prevention of venous thromboembolism (VTE). For many years, oral vitamin $\mathrm{K}$ antagonists such as warfarin have been widely prescribed to prevent embolism. Warfarin inhibits the vitamin $\mathrm{K}$ epoxide reductase complex via reducing the activities of vitamin K-dependent clotting factors II, VII, IX and $\mathrm{X}$ (1). Although warfarin has a long history of clinical use and is considered the gold standard, it has many limitations such as narrow therapeutic window, requirement of regular monitoring, and multiple drug and food interactions (1). Recently, non-vitamin K oral anticoagulant agents (NOACs) have been largely used which specifically target key molecules within the coagulation cascade. These mainly include factor Xa inhibitors (i.e. rivaroxaban, apix-

${ }^{1}$ Department of Cardiovascular Surgery, Gülhane Training and Research Hospital, Ankara, Turkey, ${ }^{2}$ Department of Biology, Faculty of Science, Hacettepe University, Ankara, Turkey, and ${ }^{3}$ Department of Statistics, Faculty of Science, Hacettepe University, Ankara, Turkey

Address for correspondence: E. Kubat, Department of Cardiovascular Surgery, Gülhane Training and Research Hospital, General Dr.Tevfik Saglam Cd. No: 106010 Etlik, Ankara, Turkey.

Phone: +90.533 .6992600$ aban) and direct thrombin inhibitors (dabigatran) (2). The European and American guidelines recommend warfarin usage for the treatment of venous thromboembolism and stroke prevention in non-valvular atrial fibrillation $(3,4,5)$. In addition, NOACs are recommended with a level of evidence similar to that of warfarin and have been introduced into the clinical practice and widely used all around the world (2). These new anticoagulants have some advantages over warfarin, such as predictable pharmacokinetics allowing a fixed-dose regimen without routine coagulation monitoring, and less drug and food interactions. However, the main disadvantage of these drugs is a lack of routinely used specific reversal agents (6).

Ximelagatran, the first NOAC, is an oral direct thrombin inhibitor which was withdrawn from the market due to liver toxicity concerns, particularly extended (up to 35 days) prophylaxis for VTE, despite its promising efficacy (7). Acute gastrointestinal (GI) bleeding represents the most common adverse event associated with the use of oral anticoagulant therapy, even when maintained within the usual therapeutic ranges (8). Due to increasing prescription of anticoagulants worldwide, GI adverse events are more common issues and challenges for clinicians. Therefore, pre-clinical and clinical studies have been conducted to compare efficacy and safety between NOACs and warfarin. However, there is no head-to-head cytotoxicity study comparing warfarin, dabigatran, rivaroxaban, and apixaban in the literature. In the present study, therefore, we aimed to compare warfarin and NOACs on 
cell culture model to make a possible correlation between cell cytotoxicity and GI side effects in the real-life setting.

\section{Materials and methods}

\section{Cell cultures and test materials}

Since clinical doses, bioavailability of drugs, and amounts of drugs remaining in the gastrointestinal lumen are not the same, the proportion of drugs which directly contact with the cells in the intraluminal side is different. However, as our goal is to investigate the cytotoxic effect of drugs that come into contact with the gastrointestinal tract prior to absorption, the concentrations of drugs were set on the basis of an equal starting amounts of drugs according to their molecular weights. Technically, L929 murine fibroblastic cells were placed in 96-well culture plates (Greiner Bio-One, Germany) at an initial density of 25,000 cells $/ \mathrm{ml}$ in six replicates and incubated in the Dulbecco's Modified Eagle's Medium (DMEM)/ Ham's F12 (Biowest Inc., Nuaillé, France) supplemented with 10 $\%$ fetal bovine serum (FBS) (Biowest Inc., Nuaillé, France) in a humidified atmosphere of $95 \%$ air and $5 \% \mathrm{CO}_{2}$ for 12 hours at $37{ }^{\circ} \mathrm{C}$. Following incubation, the cells were treated with six dilutions of the four test materials (Pradaxa (dabigatran etexilate, $150 \mathrm{mg}$ ), Xarelto (rivaroxaban, $20 \mathrm{mg}$ ), Eliquis (apixaban, $5 \mathrm{mg}$ ), and Coumadin (warfarin, $5 \mathrm{mg}$ )). Test materials were prepared in (DMEM)/Ham's F12 supplemented with $1 \%$ dimethyl sulfoxide (DMSO) (Sigma Chemical Co., St. Louis, MO), which is a safe organic solvent to prepare solutions used in cell culture tests. For each agent, stock solutions were prepared as $0.30 \mathrm{~g} / 20 \mathrm{ml}$ for the initial dose, according to the molecular weight of the agents (dabigatran: $627.746 \mathrm{~g} / \mathrm{mol}$; rivaroxaban: $435.879 \mathrm{~g} / \mathrm{mol}$; apixaban: $459.506 \mathrm{~g} / \mathrm{mol}$; warfarin: $308.333 \mathrm{~g} / \mathrm{mol}$ ). No capsule was used for capsule agents (dabigatran and apixaban), and all agents were prepared as powder and dissolved in $0.1 \%$ DMSO to obtain stock solutions. Stock solutions were kept at room temperature for 24 hours and then filtered using $0.8 \mu \mathrm{m}$ and $0.2 \mu \mathrm{m}$ filters before use. Stock solutions underwent serial dilution and were prepared in six dilutions in (DMEM)/Ham's F12 medium containing 10\% FBS. The concentrations of the test materials are shown in Table 1 . The DMEM/F12 which did not contain DMSO while containing only DMEM/F12 medium were served as control groups.

\section{Assessment of cell morphology}

Morphological changes of cells were examined under an inverting microscope (IX70 Olympus, Japan). All groups within test series were compared with the control group separately for each assessment period.

Tab. 1. Concentrations of test materials.

\begin{tabular}{lcccccc}
\hline Test Materials & \multicolumn{7}{c}{ Dilutions $(\mu \mathrm{M})$} \\
\cline { 2 - 7 } & I & II & III & IV & V & VI \\
\hline Dabigatran & 24 & 12 & 6 & 3 & 1.5 & 0.75 \\
Rivaroxaban & 30 & 15 & 7.5 & 3.75 & 1.875 & 0.975 \\
Apixaban & 30 & 15 & 7.5 & 3.75 & 1.875 & 0.975 \\
Warfarin & 50 & 25 & 12.5 & 6.25 & 3.125 & 1.5625 \\
\hline
\end{tabular}

\section{Assessment of cell viability}

The cell viability was determined by MTT assay. The viability of L929 cells were examined at 24 and 48 post-incubation hours. At each assessment period, the culture medium was removed and $100 \mu \mathrm{L}$ DMEM/F12-without FBS containing $12.5 \mu \mathrm{L}$ MTT were added into each well. Culture plates were covered with aluminum foil and cells were then incubated in the dark for four hours. At the end of the incubation period, MTT solution was removed from the wells and $100 \mu \mathrm{L}$ isopropyl alcohol was added. The absorbance at $560 \mathrm{~nm}$ was measured using an ultraviolet (UV)-visible spectrophotometer (EZ Read 400 Microplate Reader, Biochrom).

\section{Statistical analysis}

Statistical analysis was performed using the IBM SPSS version 23.0 software (IBM Corp., Armonk, NY, USA). Descriptive statistics were expressed as mean values \pm standard deviation. The Kolmogorov-Smirnov test was used for the normality test. The analysis of variance (ANOVA) was used to compare the means of more than two groups. A post-hoc test (least significant difference (LSD) or Tamhane's test) was used to examine significant differences between the groups. A p value less than 0.05 was considered statistically significant.

\section{Results}

\section{Assessment of cell morphology}

At 24-hour incubation, the cells exposed to all dilutions of dabigatran showed a significant difference compared to normal fibroblastic morphology. The cells displayed cellular alterations including nuclear condensation, rounded morphology, and cell degeneration. The excessive cell death and thus accumulation of cell debris caused the culture medium to become blurred. Figure 1a-c illustrates strong toxicity compared to the control cells (Fig. 1).

In the rivaroxaban group, a moderate cytotoxic effect was shown in dilution I at 24 hours incubation (Fig. 1d). In this dilution, the cells displayed nuclear condensation and rounded morphology. The cells in dilutions II, III, IV, V, and VI demonstrated a fibroblastic morphology (Figs 1e-f) similar to that of the control cells (Fig. 1m).

In the apixaban group, the cells showed morphology similar to that in the rivaroxaban group in dilution I at 24 hours (Fig. 1g). The cells displayed degeneration in form of nuclear condensation and rounded cell morphology. Cells in dilutions II, III, IV, V, and VI demonstrated a fibroblastic morphology (Figs 1h-i) similar to that of the control cells (Fig. 1m).

At 24 hours, the cells exposed to all dilutions of warfarin showed a morphology similar to those in the rivaroxaban and apixaban groups. A moderate cytotoxic effect was shown in dilution I at 24 hours and the cells displayed nuclear condensation and rounded morphology (Fig. 1j). The cells in dilutions II, III, IV, V, and VI demonstrated a fibroblastic morphology (Figs 1k-1) similar to that of the control cells (Fig. 1m).

At 48 hours, cells did not show significant morphological changes compared to the cells at 24 hours in all dilutions of four test materials. 

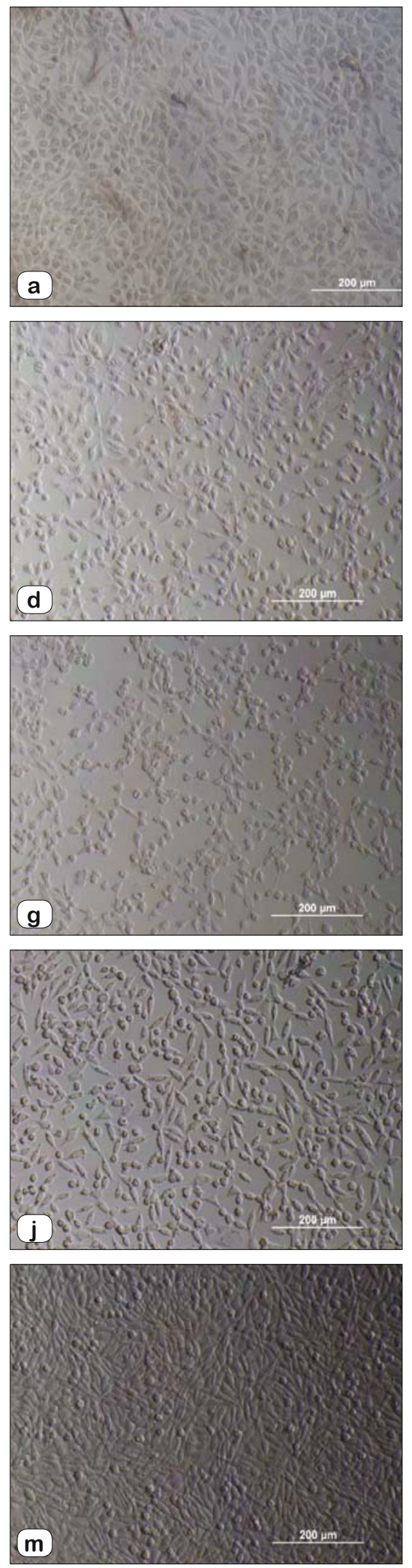
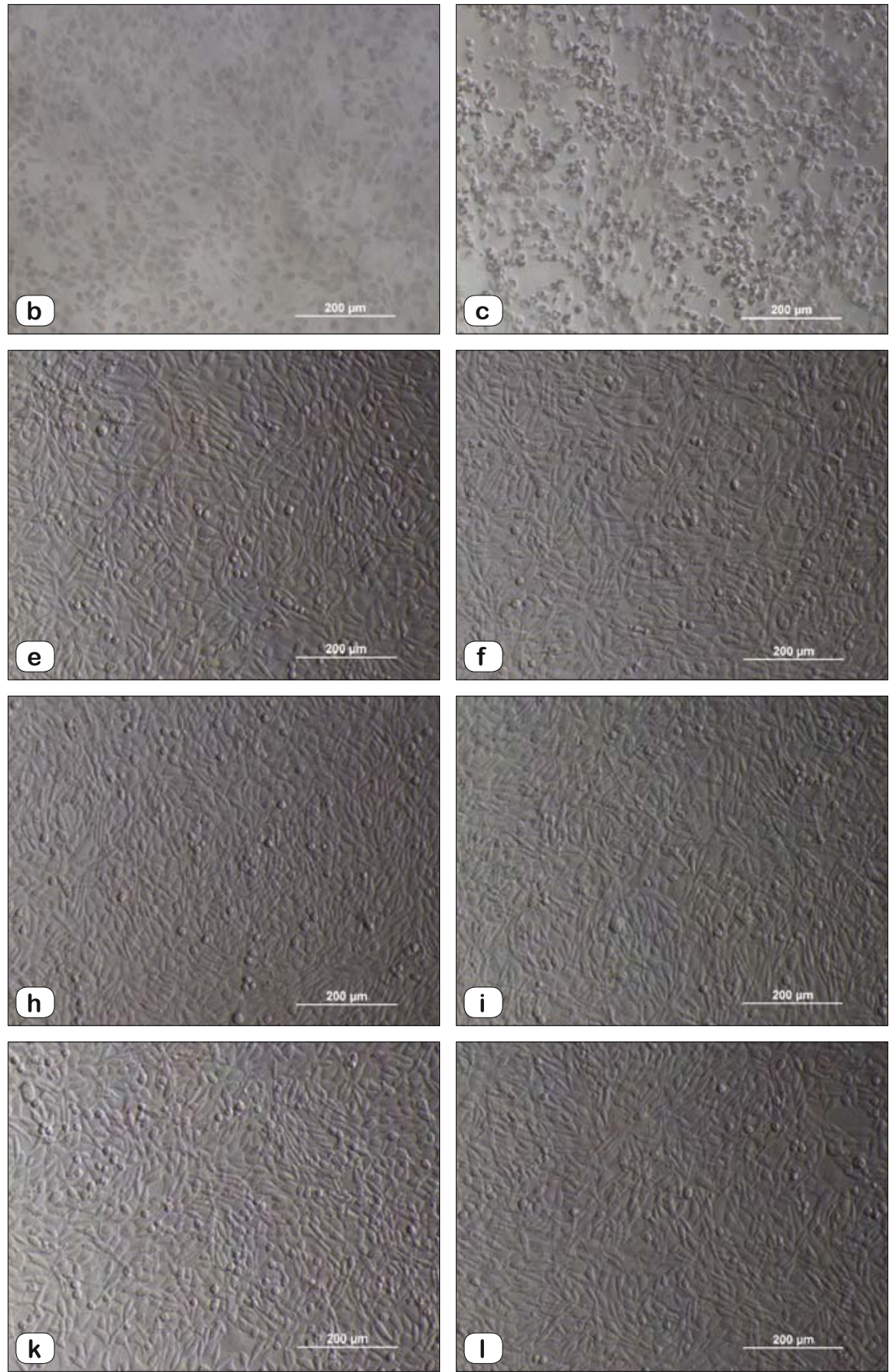

Fig. 1. Morphological appearance of L929 mouse fibroblasts exposed to Dabigatran Dilution I (a); Dilution III (b); Dilution VI (c); Rivaroxaban Dilution I (d); Dilution III (e); Dilution VI (f); Apixaban Dilution I (g); Dilution III (h); Dilution VI (i); Warfarin Dilution I (j); Dilution III (k); Dilution VI (l) and Control (m) (10×) at 24 hours of incubation. 
$24 \mathrm{~h}$

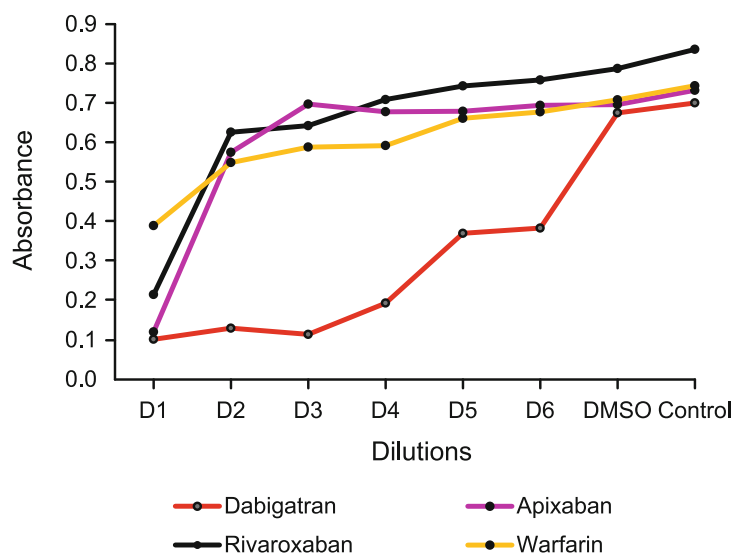

Fig. 2. Comparison of cytotoxic effects at 24 hours.

\section{Assessment of cell viability}

The cell viability was examined for each agent in different dilutions and compared with the control group at 24 and 48 hours after incubation (Figs 2 and 3). The ANOVA was performed to evaluate significant differences among all dilutions and a P value of 0.000 was obtained, indicating that the difference was statistically significant between the groups. At 24 hours, dabigatran and warfarin showed a cytotoxic effect in all dilutions, while rivaroxaban showed this effect in dilutions I, II, III, and IV while apixaban in dilution I only. These results were found to be statistically significant. At 48 hours, dabigatran, and warfarin showed cytotoxicity in all dilutions, while rivaroxaban showed this effect dilution I, II, III, while indicating statistical significance.
$48 \mathrm{~h}$

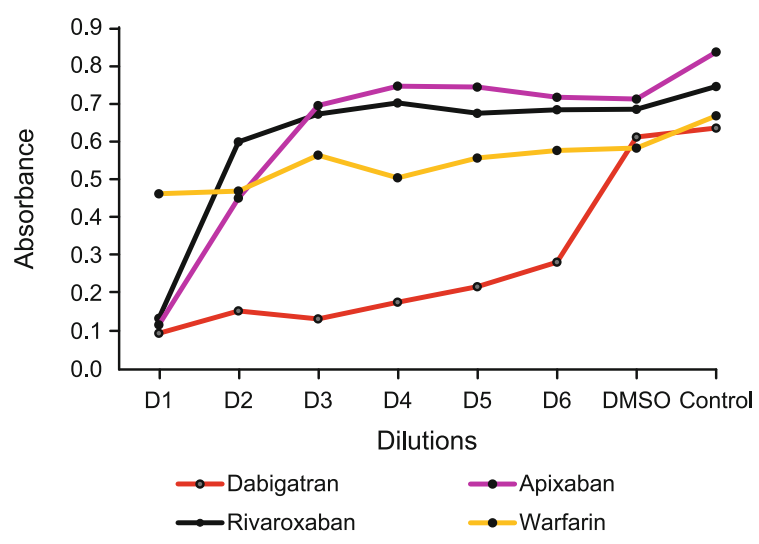

Fig. 3. Comparison of cytotoxic effects at 48 hours.

The cell viability of each agent at 24 and 48 hours in each dilution is shown in Tables $2-5$ ( $\mathrm{p}$ values added). Both morphological results and MTT analysis showed no significant difference between DMSO and control groups. In order to avoid confusion, the comparison was only made with the control group while DMSO group was excluded.

\section{Discussion}

Currently, NOACs and warfarin, which are used to prevent atrial fibrillation and venous thrombosis-related embolisms, have distinct pharmacological properties $(1,2)$. Although there is no head-to-head comparison study for NOACs, there is a consensus

Tab. 2. Cell viability of dabigatran at 24 and 48 hours in each dilution compared to control group.

\begin{tabular}{|c|c|c|c|c|c|c|c|}
\hline & & I & II & III & IV & $\mathrm{V}$ & VI \\
\hline $24 \mathrm{~h}$ & Mean \pm sd & $0.102 \pm 0.008$ & $0.13 \pm 0.022$ & $0.114 \pm 0.009$ & $0.194 \pm 0.036$ & $0.37 \pm 0.092$ & $0.384 \pm 0.080$ \\
\hline $\mathrm{D}$ & $\mathrm{p}$ & $<0.001$ & $<0.001$ & $<0.001$ & $<0.001$ & $<0.001$ & 0.001 \\
\hline $48 \mathrm{~h}$ & Mean \pm sd & $0.095 \pm 0.006$ & $0.154 \pm 0.032$ & $0.133 \pm 0.008$ & $0.177 \pm 0.024$ & $0.218 \pm 0.029$ & $0.282 \pm 0.048$ \\
\hline $\mathrm{D}$ & $\mathrm{p}$ & $<0.0$ & $<0.001$ & $<0.001$ & $<0.001$ & $<0.001$ & $<0.001$ \\
\hline
\end{tabular}

Tab. 3. Cell viability of rivaroxaban at 24 and 48 hours in each dilution compared to control group.

\begin{tabular}{|c|c|c|c|c|c|c|c|}
\hline & & I & II & III & IV & $\mathrm{V}$ & VI \\
\hline $24 \mathrm{~h}$ & Mean \pm sd & $0.215 \pm 0.091$ & $0.627 \pm 0.073$ & $0.643 \pm 0.149$ & $0.709 \pm 0.023$ & $0.744 \pm 0.064$ & $0.759 \pm 0.059$ \\
\hline $\mathrm{R}$ & $\mathrm{p}$ & $<0.001$ & $<0.001$ & 0.001 & 0.018 & 0.080 & 0.141 \\
\hline $48 \mathrm{~h}$ & Mean \pm sd & $0.135 \pm 0.021$ & $0.599 \pm 0.06$ & $0.671 \pm 0.036$ & $0.701 \pm 0.035$ & $0.674 \pm 0.129$ & $0.683 \pm 0.084$ \\
\hline $\mathrm{R}$ & $\mathrm{p}$ & $<0.001$ & 0.059 & 0.669 & 0.996 & 1.000 & 0.997 \\
\hline
\end{tabular}

sd - standard deviation, *P values compared to control group. I - Dilution I; II - Dilution II; III - Dilution III; IV - Dilution IV and V - Dilution V. R - Rivaroxaban

Tab. 4. Cell viability of apixaban at 24 and 48 hours in each dilution compared to control group.

\begin{tabular}{lcccccc}
\hline & & I & II & III & IV & V I \\
\hline $24 \mathrm{~h}$ & Mean \pm sd & $0.121 \pm 0.009$ & $0.575 \pm 0.022$ & $0.698 \pm 0.105$ & $0.678 \pm 0.178$ & $0.679 \pm 0.093$ \\
$\mathrm{~A}$ & $\mathrm{p}$ & $<0.001$ & 0.091 & 1.000 & $0.695 \pm 0.063$ & 1.000 \\
\hline $48 \mathrm{~h}$ & Mean $\pm \mathrm{sd}$ & $0.117 \pm 0.021$ & $0.451 \pm 0.059$ & $0.694 \pm 0.071$ & $0.745 \pm 0.04$ & $0.743 \pm 0.118$ \\
$\mathrm{~A}$ & $\mathrm{p}$ & $<0.001$ & $<0.001$ & 0.001 & 0.033 & 0.029 \\
\hline sd - standard deviation, *P values compared to control group. I - Dilution I; II - Dilution II; III - Dilution III; IV - Dilution IV and V - Dilution V. A - Apixaban
\end{tabular}


Tab. 5. Cell viability of warfarin at 24 and 48 hours in each dilution compared to control group.

\begin{tabular}{|c|c|c|c|c|c|c|c|}
\hline & & I & II & III & IV & $\mathrm{V}$ & VI \\
\hline $24 \mathrm{~h}$ & Mean \pm sd & $0.390 \pm 0.071$ & $0.550 \pm 0.068$ & $0.589 \pm 0.05$ & $0.593 \pm 0.049$ & $0.662 \pm 0.032$ & $0.678 \pm 0.021$ \\
\hline W & $\mathrm{p}$ & $<0.001$ & $<0.001$ & $<0.001$ & $<0.001$ & 0.006 & 0.024 \\
\hline $48 \mathrm{~h}$ & Mean \pm sd & $0.462 \pm 0.097$ & $0.469 \pm 0.042$ & $0.563 \pm 0.045$ & $0.504 \pm 0.034$ & $0.556 \pm 0.048$ & $0.576 \pm 0.062$ \\
\hline $\mathrm{W}$ & $p$ & $<0.001$ & $<0.001$ & 0.004 & $<0.001$ & 0.002 & 0.011 \\
\hline
\end{tabular}

on the efficacy and safety of NOACs, as warfarin is one of the commonly used oral anticoagulants $(9,10,11,12)$. In the studies, the primary efficacy outcome was evaluated relative to the presence of systemic or thromboembolic events, while primary safety outcomes were relative to major bleeding including GI bleeding and intracranial bleeding. Although these studies provide us with important clinical information, preclinical, and clinical studies are still ongoing to obtain further information about NOACs. In our study, the in vitro cytotoxic effects of four agents were compared in cell culture to investigate the possible toxic effects of the agents which are in contact with GI tract before absorption.

The GI tract is a long, twisting tube starting at the mouth and ending at the anus. It exerts secretory, absorptive, preservative and combined effects to maintain the functions of the GI system (13). These functions are supplied by rich mucosal and submucosal vessel network and strong blood circulation (13). However, drug use and infections may lead to mucosal damage in the GI tract and eventually to bleeding (13). In a study reviewing the efficacy and safety data of anticoagulants in a real-life setting, each NOAC was found to have a unique profile when compared to warfarin in terms of major bleeding, intracranial bleeding, and GI bleeding (Yao et al, 2013). Although the incidence of rivaroxaban-induced intracranial bleeding was lower than that induced by warfarin, the incidence of GI bleeding was identical to that induced by warfarin. Moreover, while the incidence rates of intracranial and GI bleeding induced by dabigatran were identical to those induced by warfarin, the incidence rates of apixaban-induced intracranial and GI bleeding were lower than those induced by warfarin $(3,4$, $9,10,11,12)$. Therefore, there is no sole factor that could explain the GI side effect profile of anticoagulant agents.

In the literature, four hypotheses have been proposed to explain the underlying GI bleeding mechanism of oral anticoagulants: topical biological effect which is not related to coagulation, systemic anticoagulant effect, topical anticoagulant effect, and direct topical cytotoxic effect of the drug (15). The GI side effects of anticoagulants may be explained by one or all of these factors (15). Non-steroidal anti-inflammatory drugs (in particular, acetylsalicylic acid) cause mucosal toxicity due to decreased prostaglandin concentration in the gastroduodenal mucosa. It then modulates the biological activity, thereby disrupting the mucosal healing and protection. However, there is no available data in the literature regarding the topical biological effect of anticoagulant agents like acetylsalicylic acid on the GI mucosal barrier. Oral agents which are associated with direct topical cytotoxic effects on the GI system may result in GI intolerance and GI bleeding. Previous studies using oral anticoagulants showed that the with- drawal rate due to GI intolerance was higher with dabigatran when compared to warfarin $(10,16)$. Also, according to the prescribing information of NOACs and warfarin, dabigatran and rivaroxaban, but not apixaban, are associated with dyspepsia $(17,18,19)$. Dabigatran-related dyspeptic symptoms have been suggested to result from the hyperacidic microenvironment on the surface of gastric mucosa formed by the tartaric acid within the galenic formation to increase the absorption, but not from the cytotoxic effects of the drug (20). Also, several studies using rivaroxaban, apixaban, warfarin and warfarin-derivatives have demonstrated that these drugs prevent cellular proliferation and migration in certain cell lines and show anti-tumorigenic or apoptotic effect at certain doses $(21,22,23)$. However, there is no study in the literature which investigates the direct cytotoxic effects of these drugs. Therefore, in the present study, we investigated direct cytotoxic effects of these four agents in different dilutions. We interpreted our study results with documented GI side effects. Thus, we integrated our in vitro results into the clinical practice.

In the present study, the morphological analysis revealed that dabigatran showed cytotoxic effects in all dilutions at 24 hours, while rivaroxaban, apixaban, and warfarin had similar results with DMSO and control group, except for dilution I. According to the MTT analysis, dabigatran showed toxic effects in all dilutions with the highest cytotoxicity. In a cytotoxicity study, although the study design and test concentrations of dabigatran were different from our study, no cytotoxic effect was shown with dabigatran and degradation products (24). In a real-life setting and clinical practice, mid-esophageal ulcer was reported in some of the patients using dabigatran, suggesting that dabigatran has a direct caustic effect on capsular formation $(25,26)$. In the present study, although warfarin showed similar fibroblastic morphology with the control group, except for dilution I, cell viability was found to be statistically significantly lower in all dilutions, compared to the control group. Despite its statistical significance, this finding suggests that dabigatran may be associated with dyspeptic symptoms as highly as warfarin (10). In our study, cell viability was higher with rivaroxaban, compared to dabigatran and warfarin, except for dilution I; however, apixaban was found to be the least cytotoxic agent at 24 hours among all agents, compared to the control group.

There is a further proposal regarding the clinical discrepancy of GI bleeding side effects of anticoagulants: the amount of nonabsorbable drug left in the intraluminal region may exert topical anticoagulant effects (15). In addition, the absorption site is a critical factor which influences the intraluminal amount of the oral agents, since the surface contact ratio of the agent increases 
in the GI system, based on the absorption site. Bioavailability of warfarin reaches up to $100 \%$, and therefore, it may not show this effect due to inactive intraluminal warfarin or warfarin metabolites. In addition, although the absorption site is unclear $(1,27)$, it is reported that warfarin was mainly absorbed in the proximal intestines. Therefore, GI bleeding rate induced by warfarin may be explained by the systemic anticoagulant effect of the agent (17). Although it is a pro-drug, inactive dabigatran can be activated in the lumen due to intraluminal bacterial esterases (15). Rivaroxaban is an active drug and its bioavailability is dose-dependent. The absolute bioavailability of rivaroxaban $20 \mathrm{mg}$ is $66 \%$ and it reaches up to $75 \%$ with meals. It is absorbed in the stomach through the GI system (18). Apixaban is also an active drug with a bioavailability of $50 \%$. It is absorbed through the GI system and $55 \%$ of the drug is absorbed in the distal ileum and proximal colon (19). According to the absorption sites, apixaban and rivaroxaban can be considered the main active agents for the GI tract; however, due to low bioavailability and active formation with bacterial esterases, dabigatran is the main anticoagulant which remains in the intestinal epithelium for the longest period of time. Considering these two factors, differently from warfarin, NOACs have active agents in the intraluminal region. Therefore, in addition to systemic anticoagulant effects, topical anticoagulant effects of NOACs may play a role in the mechanism of GI bleeding, similar to warfarin, although the exposure duration of cytotoxic effects of NOACs in the GI tract epithelium is longer than that of warfarin. To test this hypothesis, in the present study, we created an in vitro cell culture model. At 48 hours, dabigatran was found to be the main cytotoxic agent with associated impairment of cell morphology in all dilutions and low cell viability. Under inverted microscope, there was no significant difference in the cell morphology in cultures treated with warfarin, apixaban, and rivaroxaban at 24 and 48 hours; however, rivaroxaban was the least cytotoxic agent in terms of cell viability compared to the control group. In previous studies comparing NOACs with warfarin, the rate of GI bleeding associated with dabigatran and rivaroxaban were found to be similar to that associated with warfarin, while apixaban was associated with a lower rate of GI bleeding compared to warfarin $(3,4,9,10,11,12)$. In addition, a randomized-controlled study comparing dabigatran and warfarin showed that dabigatran and warfarin had similar GI bleeding rate, although the rate of lower GI bleeding and the incidence of GI bleeding in elderly (>75 years) were higher with dabigatran, compared to warfarin (28). In elderly, as the GI motility is slower, dabigatran is considered to stay in the GI tract, thereby, leading to increased bleeding. The relatively lower GI bleeding rate of dabigatran can be explained by the facts that its intraluminal exposure duration is the longest, it is activated with bacterial esterases, and it is the most cytotoxic agent in our study. Rivaroxaban showed less cytotoxicity at 48 hours compared to apixaban. However, apixaban has a low bleeding profile compared to rivaroxaban. This can be explained by the low amount of unabsorbed apixaban in the intraluminal surface when compared to rivaroxaban.

There were several limitations in the present study. Firstly, gastrointestinal epithelial cell line was not used in the study. Cy- totoxic effects of drugs were investigated in L929 mouse fibroblast cell line which is commonly used and gives decisive results in cytotoxicity tests. Next, the drugs were performed in a cell culture milieu that contained $10 \%$ fetal bovine serum which induces cellular proliferation. This may explain the increase in cell number at 48 hours of incubation period.

In conclusion, in the present study, we used L929 mouse fibroblast cell line due to its high reproducibility rate and reliable results in the cytotoxicity tests, to investigate cytotoxic effects of NOACs and warfarin, in an in vitro model. The results of present study suggest that the GI side effect profile of these four agents in real life is consistent with the results obtained from the clinical studies. It is thought that the tartaric acid within the galenic formation of dabigatran and anticoagulant effect of warfarin are responsible for major GI side effects, but not for the cytotoxic effects of the drugs. Our results showed that dabigatran was the most cytotoxic agent affecting both morphology and cell viability at 24 and 48 hours. Apixaban and rivaroxaban were the least cytotoxic agents at 24 hours, respectively. Warfarin was found to be the second most cytotoxic agent at both time points. We believe that direct cytotoxic effects of these drugs call for a preliminary consideration for gastrointestinal side effects. We also believe that the present study could contribute to further scientific studies affecting the modification of the structure or dose of these drugs in clinical practice in an effort to minimize their gastrointestinal side effects, shed light into the primary safety issues and determine anticoagulant cytotoxicity.

\section{References}

1. Jones DR, Miller GP. Assays and applications in warfarin metabolism: what we know, how we know it and what we need to know. Expert Opin Drug Metab Toxicol 2011; 7: 857-874.

2. Di Minno A, Frigerio B, Spadarella G, Ravani A, Sansaro D, Amato M, Kitzmiller JP et al. Old and new oral anticoagulants: Food, herbal medicines and drug interactions. Blood Rev 2017; 31: 193-203.

3. Kirchhof P, Benussi S, Kotecha D, Ahlsson A, Atar D, Casadei B, Castella M et al. ESC Guidelines for the management of atrial fibrillation developed in collaboration with EACTS. Europace 2016; 18: 1609-1678.

4. January CT, Wann LS, Alpert JS, Calkins H, Cigarroa JE, Cleveland JC Jr et al. Field ME. 2014 AHA/ACC/HRS guideline for the management of patients with atrial fibrillation: a report of the American College of Cardiology/American Heart Association Task Force on Practice Guidelines and the Heart Rhythm Society. JACC 2014; 64: 2305-2307.

5. Kearon C, Akl EA, Ornelas J, Blaivas A, Jimenez D, Bounameaux H, Huisman M et al. Antithrombotic therapy for VTE disease: CHEST guideline and expert panel report. Chest 2016; 149: 315-352.

6. Sousa-Uva M, Head SJ, Milojevic M, Collet JP, Landoni G, Castella $\mathbf{M}$ et al. Guidelines on perioperative medication in adult cardiac surgery. Eur J Cardiothorac Surg 2018; 53: 5-33.

7. Keisu M, Andersson TB. Drug-induced liver injury in humans: the case of ximelagatran. Handb Exp Pharm 2010; 196: 407-418.

8. Hippisley-Cox J, Coupland C. Predicting risk of upper gastrointestinal bleed andintracranial bleed with anticoagulants: cohort study to derive a validate QBleedscores. Brit Med J 2014; 349: 1-21. 
706-712

9. Granger CB, Alexander JH, McMurray JJ, Lopes RD, Hylek EM, Hanna $\mathbf{M}$ et al. Apixaban versus warfarin in patients with atrial fibrillation. NEJM 2011; 365: 981-992.

10. Connolly SJ, Ezekowitz MD, Yusuf S, Eikelboom J, Oldgren J, Parekh A, Pogue et al. Dabigatran versus warfarin in patients with atrial fibrillation. NEJM 2009; 361: 1139-1151.

11. Patel MR, Mahaffey KW, Garg J, Pan G, Singer DE, Hacke W, Breithardt G et al. Rivaroxaban versus warfarin in nonvalvular atrial fibrillation. NEJM 2011; 365: 883-891.

12. Schulman S, Kearon C, Kakkar AK, Mismetti P, Schellong S, Eriksson H, Baanstra D, Schnee J, Goldhaber SZ (2009). Dabigatran versus warfarin in the treatment of acute venous thromboembolism. NEJM 361: $2342-2352$.

13. Young B, O'Dowd, Woodford P. Gastrointestinal Tract. Wheater's Functional Histology. 6th ed. Phildelphia, PA, USA: Churchill Livingstone Elsevier 2013.

14. Yao X, Abraham NS, Sangaralingham LR, Bellolio MF, McBane RD, Shah ND, Noseworthy PA. Effectiveness and Safety of Dabigatran, Rivaroxaban, and Apixaban Versus Warfarin in Nonvalvular Atrial Fibrillation. JAHA 2016; 13: 1-18.

15. Desai J, Kolb JM, Weitz JI, Aisenberg J. Gastrointestinal bleeding with the new oral anticoagulants-defining the issues and the management strategies. Thromb Haemost 2013; 110: 205-212.

16. Staerk L, Gislason GH, Lip GY, Fosbøl EL, Hansen ML, Lamberts M, Bonde AN et al. Risk of gastrointestinal adverse effects of dabigatran compared with warfarin among patients with atrial fibrillation: a nationwide cohort study. Europace 2015; 17: 1215-1222.

17. Stangier J, Rathgen K, Stähle H, Gansser D, Roth W. The pharmacokinetics, pharmacodynamics and tolerability of dabigatran etexilate, a new oral direct thrombin inhibitor, in healthy male subjects. Br J Clin Pharmacol 2007; 64: 292-303.
18. Gulseth MP, Michaud J, Nutescu EA. Rivaroxaban: an oral direct inhibitor of factor Xa. Am J Health Syst Pharm 2008; 15: 1520-1529.

19. Raghavan N, Frost CE, Yu Z, He K, Zhang H, Humphreys WG, Pinto $\mathbf{D}$ et al. Apixaban metabolism and pharmacokinetics after oral administration to humans. Drug Metab Dispos 2009; 37: 74-81.

20. Hoffman A, Galle PR. Gastrointestinal disorders and dabigatran. Scand J Gastroentero 2012; 48: 9-16.

21. Guasti L, Squizzato A, Moretto P, Vigetti D, Ageno W, Dentali F, Maresca AM et al. In vitro effects of Apixaban on 5 different cancer cell lines. PLoS One 2017; 12: 1-19.

22. Emami S, Dadashpour S. Current developments of coumarin-based anti-cancer agents in medicinal chemistry. Eur J Med Chem 2015; 102 : 611-630.

23. Núñez-Navarro NE, Segovia FG, Burgos RA., Lagos CF, FuentesIbacache N, Faúndez MA, Zacconi, FC. Microwave Assisted Synthesis of Novel Six-Membered 4-C, 4-O and 4-S Lactams Derivatives: Characterization and in vitro Biological Evaluation of Cytotoxicity and Anticoagulant Activity. JBCS 2017; 28: 203-207.

24. Bernardi RM, D’Avila FB, Todeschini V, Andrade JM, Fröehlich PE, Bergold AM. Main Degradation Products of Dabigatran Etexilate Evaluated by LC-UV and LC-ESI-MS, Degradation Kinetics and in vitro Cytotoxicity Studies. JBCS 2017; 26: 660-666.

25. Wood M, Shaw P. Pradaxa-induced esophageal ulcer. BMJ Case Reports 2015; bcr2015211371.

26. Fujikawa K, Takasugi N, Goto T, Minatoguchi S. Very Late-Onset Dabigatran-Induced Esophageal Injury. Can J Cardiol 2017; 33: 554-555.

27. Sabol BJ, Basa RR, Wilkins CE. Malabsorption-associated warfarin resistance. Am J Health-Syst Ph 2009; 66: 1548-1553.

28. Kumar R, Rahman AM, Henry BL. A Review of the Clinical Subgroup Analyses from the RE-LY Trial. Rev Cardiovasc Med 2016; 17 : $40-48$. 